\title{
Comment
}

\section{The socialisation of scientific and technological research}

\begin{abstract}
In the last decades, production of science and technology as well as science-society relationships started changing rapidly. Research is asked to be more effective, fast, accountable, trans-disciplinary, result-oriented, policy-driven and able to generate benefits for people and firms in the short and middle run. While a strong intensification of science-society relationships is occurring, an increasing number of actors and stakeholders are involved in research production. At the same time, pervasiveness of technology is rendering users an active part in technological development; economic and social interests on science and technology are growing on a global scale; new democratic and ethical issues emerge. Despite the European institutions' efforts, all those trends and phenomena are occurring in an extremely fragmented way. In this scenario, a fairly balanced and consistent co-evolution between science and society can no longer be taken for granted.

This is just the starting point of the following comment section that, through the Luciano d'Andrea, Sally Wyatt, Erik Aarden, Jos Lejten and Peter Sekloča's writings, aims to analyse the different aspects and questions around the socialisation of science and technology's matter.
\end{abstract}

In the last decades, modes of production of science and technology as well as science-society relationships started changing rapidly, under the pressure of broader trends of transformation affecting contemporary societies. Research is asked to be more effective, fast, accountable, trans-disciplinary, result-oriented, policy-driven and able to generate benefits for people and firms in the short and middle run. At the same time, a strong intensification of science-society relationships is occurring, at multiple levels, without authorities or "traffic lights" able to regulate them; an increasing number of actors and stakeholders are involved in research production; pervasiveness of technology is rendering users an active part in technological development; economic and social interests on science and technology are growing on a global scale; new democratic and ethical issues emerge. Moreover, at least in Europe, all that is occurring in an extremely fragmented way, despite the efforts made by European institutions in coordinating research policies and enhancing competitiveness and social acceptance of science. In this scenario, a fairly balanced and consistent co-evolution between science and society can no longer be taken for granted.

This is the starting point of the following comments, revolving around the question of the socialisation of science and technology. As argued by Luciano d'Andrea in his contribution, the application of the notion of socialisation to a social institution like scientific and technological research, rather than to individuals, could be helpful to better understand this complex set of transformations, putting in the forefront what is at stake with them, that is the risk of a decreasing embeddedness of science in society and, therefore, a decreasing capacity of scientific and technological research to adapt to a rapidly changing society. Hence the paradox of a science which is intrinsically strong, in terms of results attained, but which growingly appears to be socially weak. The proposed analytical frame, recently tested in the European project "Social Sciences and the European Research Capacities (SSERC) can be also understood as an attempt to restore a unitary view of the many and often problematic phenomena of a social nature incorporated into scientific and technological research, presently studied and dealt with under different headings (innovation, evaluation, scientific practices, and the like) and by different specialised communities of researchers, experts, professionals and policy makers.

On the other hand, as stressed by Sally Wyatt, socialisation - both when the concept is applied to individuals and when it is applied to social institutions - refers anyhow to a process of transaction between individuals and society. Therefore, socialisation cannot be viewed as a one-way process, where individuals are fully subjected to the institutions or vice-versa, but as a process of balancing based on mutual interactions. Consequently, besides a risk of "hypo-socialisation", there is also a possible risk of "over-socialising" science, endangering its peculiar features and impeding to valuable elements characterising science to be transferred and shared by other social institutions. 
According to Erik Aarden, a reflection on socialisation cannot ignore the strong interconnectedness between science and cultures, which arrives to affect the most intimate structures of scientific discovery. As things stand, socialisation of science and technology cannot be viewed as a unique, unambiguous project to be carried out on the basis of predetermined recipes. Rather, it cannot be but a complex social process involving a large array of actors, allowing to develop socialisation strategies fully embedded in technological and political culture and seriously taking into account cultural approaches to technoscience, national identity and political decision-making.

In his comment, Jos Leijten opens a window on the possible future developments of socialisation processes. Some recent trends in the most promising technological sectors (bio-technology, nanotechnology) suggest that, in the future, final users will play an increasing role in developing new technologies and new applications, having a growing possibility to access tools allowing a direct manipulation of the basic building blocks (genes, atoms, etc.), as already happened - thanks to the development of powerful personal computers - in the case of information. In this environment, socialisation scenarios will become richer, but more complex too, requiring higher levels of coordination, stronger interactions among a hugely increased number of different actors, more sophisticated decision making processes, more complex behaviours and more rapid spread of shared preferences, visions and cultures.

Needless to say, also scientific communication is fully involved in science and technology socialisation. This issue is dealt with in the contributions by Peter Sekloča, Ernest Ženko and Blaž Lenarčič, who focus the attention on how the concept of "public" should be interpreted in a socialisation perspective. According to the authors, defining a clearcut boundary between science and the rest of society and therefore between scientists and the public is misleading. It is obvious that the public is not involved in evaluating the validity of scientific results. But when all the other questions pertaining to scientific research are concerned, scientists represent only a specific group, bearing specific interests and points of view, like any other group is. Therefore, the idea of public communication about science should be understood, not as a debate between scientists and the others, but as an open discussion involving all the citizens, including scientists as citizens too. 